\title{
Tendências da morbimortalidade por câncer infantojuvenil em um polo de fruticultura irrigada
}

\author{
Trends in morbidity and mortality due to child and \\ adolescent cancer in a center of irrigated fruitculture
}

\author{
Marília Gabriella Pinheiro Silva ${ }^{1}$, Cheila Nataly Galindo Bedor ${ }^{1}$, \\ Kamilla Maria de Souza Aires Alencar², Maria Paula Curado ${ }^{3,4}$, \\ Luiza Taciana Rodrigues de Moura ${ }^{5}$
}

\begin{abstract}
Resumo
Objetivo: Descrever as tendências de morbimortalidade por câncer em jovens de 0 a 19 anos nos municípios de Petrolina (PE) e Juazeiro (BA). Método: Série histórica de morbimortalidade por câncer infantojuvenil dos residentes em Petrolina (PE) e Juazeiro (BA), no período de 2004 a 2013, usando dados do Sistema de Informação de Internação Hospitalar e Sistema de Informação de Mortalidade. As taxas de internação e mortalidade foram padronizadas por idade, tendo sido realizada análise de tendências pelo modelo de regressão Joinpoint. Resultados: As taxas de internação e de mortalidade foram mais altas em Petrolina comparadas com as de Juazeiro. Taxas de mortalidade acima de 60/1.000.0000 em ambos os municípios encontram-se acima das taxas de mortalidade brasileiras $(40,28 / 1.000 .000)$ e das taxas de mortalidade do Nordeste $(35,62 / 1.000 .000)$ para o período de 2001 a 2005. Conclusão: Houve tendência de aumento significante das taxas de internação e aumento sem significância estatística das taxas de mortalidade nos dois municípios, o que pode estar relacionado à melhoria no acesso ao serviço especializado de oncologia pediátrica, ao diagnóstico tardio e à exposição ambiental a agrotóxicos.
\end{abstract}

Palavras-chave: neoplasias; criança; adolescente; mortalidade; morbidade.

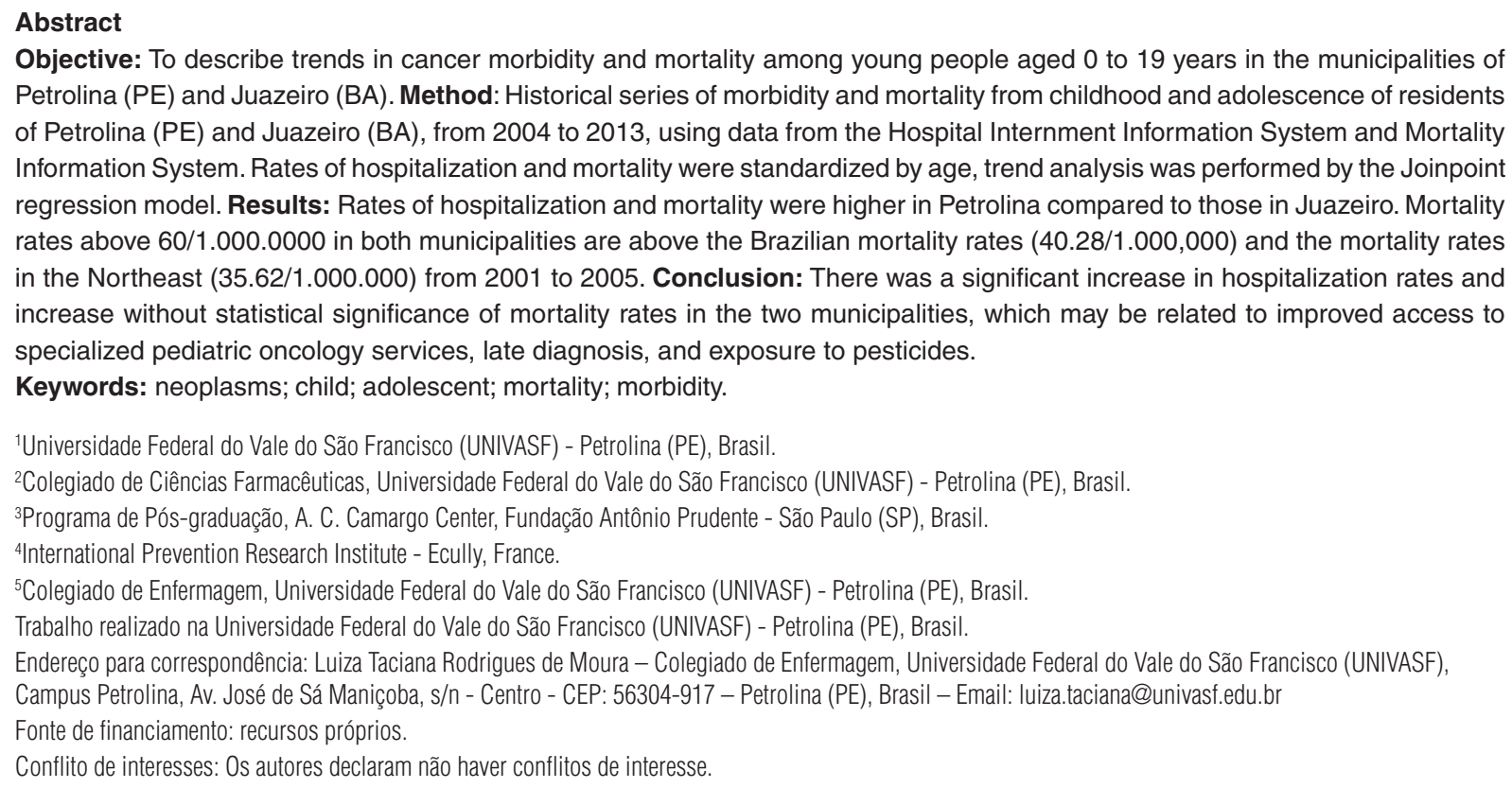
Petrolina (PE) and Juazeiro (BA). Method: Historical series of morbidity and mortality from childhood and adolescence of residents of Petrolina (PE) and Juazeiro (BA), from 2004 to 2013, using data from the Hospital Internment Information System and Mortality Information System. Rates of hospitalization and mortality were standardized by age, trend analysis was performed by the Joinpoint regression model. Results: Rates of hospitalization and mortality were higher in Petrolina compared to those in Juazeiro. Mortality rates above 60/1.000.0000 in both municipalities are above the Brazilian mortality rates $(40.28 / 1.000,000)$ and the mortality rates in the Northeast (35.62/1.000.000) from 2001 to 2005. Conclusion: There was a significant increase in hospitalization rates and increase without statistical significance of mortality rates in the two municipalities, which may be related to improved access to specialized pediatric oncology services, late diagnosis, and exposure to pesticides.

Keywords: neoplasms; child; adolescent; mortality; morbidity.

${ }^{1}$ Universidade Federal do Vale do São Francisco (UNIVASF) - Petrolina (PE), Brasil.

${ }^{2}$ Colegiado de Ciências Farmacêuticas, Universidade Federal do Vale do São Francisco (UNIVASF) - Petrolina (PE), Brasil.

${ }^{3}$ Programa de Pós-graduação, A. C. Camargo Center, Fundação Antônio Prudente - São Paulo (SP), Brasil.

${ }^{4}$ International Prevention Research Institute - Ecully, France.

${ }^{5}$ Colegiado de Enfermagem, Universidade Federal do Vale do São Francisco (UNIVASF) - Petrolina (PE), Brasil.

Trabalho realizado na Universidade Federal do Vale do São Francisco (UNIVASF) - Petrolina (PE), Brasil.

Endereço para correspondência: Luiza Taciana Rodrigues de Moura - Colegiado de Enfermagem, Universidade Federal do Vale do São Francisco (UNIVASF),

Campus Petrolina, Av. José de Sá Maniçoba, s/n - Centro - CEP: 56304-917 - Petrolina (PE), Brasil - Email: luiza.taciana@univasf.edu.br

Fonte de financiamento: recursos próprios.

Conflito de interesses: Os autores declaram não haver conflitos de interesse. 


\section{INTRODUÇÃO}

O câncer infantojuvenil, que acomete indivíduos de 0 a 19 anos de idade, pode ocorrer em qualquer local do organismo. As neoplasias mais incidentes nessa faixa etária são leucemias, linfomas, tumores do sistema nervoso central (SNC) e do sistema simpático (neuroblastomas), rabdomiossarcomas, tumor de Wilms, retinoblastomas e tumores ósseos ${ }^{1,2}$.

Poucos fatores de risco são bem estabelecidos como determinantes da maior incidência de câncer nesse grupo etário; a exposição precoce, desde a vida intrauterina, a carcinogênicos químicos como o benzeno e à radiação ionizante tem sido associada ao desenvolvimento de leucemia, o câncer mais comum na infância ${ }^{3,4}$. Estudos preliminares têm discutido a influência dos fatores genéticos como determinantes para maior incidência de osteossarcomas, tumores de células germinativas e do sarcoma de Ewing 5 .

O câncer é uma das principais causas de mortalidade em crianças de países desenvolvidos e constitui-se em uma importante preocupação em saúde pública devido aos impactos físicos, psicológicos, sociais e econômicos que atingem as crianças e seus familiares ${ }^{6}$. Nos Estados Unidos é a segunda causa de mortalidade em crianças de 0 a 14 anos, superado apenas pelas causas externas. Os tipos de neoplasias infantojuvenis mais incidentes nesse país são leucemias, tumores do SNC, neuroblastomas, tumores renais e linfomas de Hodgkin e não-Hodgkin ${ }^{7}$.

Nos países em desenvolvimento, onde a população infantil representa cerca de $50 \%$, a proporção do câncer infantil representa de $3 \%$ a $10 \%$ do total de neoplasias. Já nos países desenvolvidos, essa proporção diminui, chegando a $1 \%$. Em relação à mortalidade, nos países desenvolvidos, o óbito por neoplasia corresponde a $4 \%$ a $5 \%$ (crianças de 0 a 14 anos), e nos países em desenvolvimento, essa proporção é de $1 \%$, porque as doenças infecciosas ainda são as principais causas de óbito nessa faixa etária ${ }^{8}$.

De acordo com os dados do Instituto Nacional do Câncer (Inca), no Brasil, o percentual mediano de incidência dos tumores pediátricos é de 3\% do total das neoplasias². Em 2014, ocorreram 2.867 óbitos por câncer em crianças e adolescentes (de 0 a 19 anos), correspondendo a 3,8\% do total de mortes. No mesmo ano, essas neoplasias ocuparam a segunda posição, $7,3 \%$ das causas de morte na faixa etária de 0 a 19 anos, perdendo somente para as causas externas. Houve redução da mortalidade por causas relacionadas à prevenção por imunização e à melhora das condições de vida8.

Dadas as dificuldades no estabelecimento dos fatores de risco e a magnitude do problema, a redução da mortalidade está associada à ampliação da oferta de tratamento, detecção precoce, diagnóstico correto, tratamento em tempo hábil e minimização dos fatores de risco conhecidos ${ }^{10}$.
Os cânceres infantojuvenis têm períodos de latência curtos, desenvolvem-se rapidamente, são mais invasivos, porém mais responsivos ao tratamento do que o câncer em adultos ${ }^{11}$. Nesse sentido, o diagnóstico precoce é fundamental para reduzir as complicações e diminuir a mortalidade. Uma revisão sistemática identificou que os principais fatores relacionados ao atraso no diagnóstico foram a idade da criança no momento do diagnóstico, a topografia, o estágio do câncer e a primeira especialidade médica consultada ${ }^{6}$.

Considerando a expressão da mortalidade por câncer infantojuvenil, o conhecimento do perfil de morbimortalidade por neoplasias nessa faixa etária é fundamental para a definição e a implantação de estratégias em saúde pública que possam promover o diagnóstico precoce, como educação para a comunidade em geral a respeito do câncer, reorganização das redes de atenção à saúde, treinamento de pediatras para o reconhecimento precoce e suspeição de câncer e encaminhamento adequado aos centros de referência ${ }^{5,8,11}$.

Na região de Petrolina (PE) e Juazeiro (BA), a fruticultura irrigada fomentou um grande dinamismo na economia e na estrutura urbana, tornando-se o aglomerado urbano mais próspero do Vale do São Francisco ${ }^{12}$. Entretanto, nessa atividade agrícola, ocorre uso indiscriminado de agrotóxicos, com ênfase nos organofosforados, que interferem na saúde dos expostos ${ }^{13}$.

Nesse polo de fruticultura, estudos identificaram um aumento de mortalidade por doenças neoplásicas, em ambos os sexos, e em trabalhadores rurais as principais taxas de mortalidade por câncer estavam relacionadas às neoplasias malignas de próstata e de pulmão ${ }^{14,15}$. Em relação à morbidade, outro estudo realizado em um centro de oncologia da região descreveu as neoplasias do sistema hematológico (leucemias, linfomas e mielomas) como as mais incidentes na população estudada, sendo os trabalhadores rurais a categoria profissional mais acometida, fato que pode estar relacionado à exposição a agrotóxicos ${ }^{16}$.

Porém, nessa região, ainda não existem estudos sobre o perfil de morbimortalidade por câncer infantojuvenil, o que torna relevante a realização deste estudo. Além disso, o serviço de oncologia pediátrica foi implantado em 2011, o que reforça a necessidade de conhecer esse perfil, para uma melhor organização dos serviços de saúde locais ${ }^{17}$. O objetivo é proporcionar acesso ao diagnóstico precoce e tratamento adequado, com redução de complicações e mortalidade nessa população de risco.

Diante disso, este estudo tem o objetivo de descrever as tendências de morbimortalidade por câncer em jovens de 0 a 19 anos nos municípios de Petrolina (PE) e Juazeiro (BA), que constituem o principal polo de irrigação do Vale do São Francisco.

\section{MÉTODO}

Estudo quantitativo, ecológico e retrospectivo realizado por meio da coleta de dados secundários contidos no Sistema de Informação de Internação Hospitalar (SIH) e Sistema 
de Informação de Mortalidade (SIM) do Departamento de Informática do Sistema Único de Saúde (Datasus). A coleta de dados foi feita considerando o período de dez anos (2004-2013).

A avaliação da morbidade hospitalar foi realizada com a coleta de dados referentes às internações por neoplasias (Capítulo II da Classificação Internacional de Doenças - CID-10) ${ }^{2} \mathrm{em}$ menores de 19 anos, residentes nos municípios de Petrolina e Juazeiro. Já para avaliação da mortalidade, foram coletados os dados referentes aos óbitos por neoplasias (Capítulo II da Classificação Internacional de Doenças - CID-10) ${ }^{2}$ em menores de 19 anos, residentes nos municípios de Petrolina e Juazeiro.

Foi calculada a taxa de internação por neoplasias (Fórmula 1) e construída uma série histórica de dez anos de ambos os municípios (as taxas foram padronizadas pelo método direto, conforme Fórmula 2, considerando a população padrão mundial proposta por Segi) ${ }^{18}$. Calcularam-se as frequências relativas e absolutas de internações por câncer por faixa etária e sexo mais acometidos na população estudada. A fórmula para o cálculo da taxa de internação foi a seguinte:

$$
\begin{aligned}
& \text { Taxa de internação por neoplasias }= \frac{\mathrm{n}^{\circ} \text { de internações por neoplasias }}{\text { População de } 0 \text { a } 19 \text { anos no período }} \\
& \text { Taxa de Internação Padronizada }=\frac{(\text { população padrão na faixa etária })}{\sum(\text { população padrão }) \text { 0-19 anos }}
\end{aligned}
$$

A taxa de mortalidade bruta foi calculada de acordo com a Fórmula 3 e padronizada pelo método direto (Fórmula 4), considerando a população do Brasil conforme censo e estimativas do Instituto Brasileiro de Geografia e Estatística (IBGE) ${ }^{2}$ no período. No cálculo da taxa padronizada pela população padrão mundial, foi usada a população de Segi ${ }^{18}$. Calcularam-se também as frequências relativas e absolutas de óbitos por câncer, considerando-se a faixa etária e o sexo mais acometidos na população estudada. Utilizaram-se as seguintes fórmulas:

$$
\text { Taxa de Mortalidade bruta por neoplasias }=\frac{\begin{array}{l}
\mathrm{n}^{\circ} \text { de óbitos por neoplasias } \\
(0 \text { a } 19 \text { anos }) \times 1.000 .000
\end{array}}{\text { População de } 0 \text { a } 19 \text { anos no período }}
$$

$$
\begin{aligned}
& \left.=\sum \text { (taxa específica por idade }\right) \mathrm{x} \\
& \text { Taxa de Mortalidade Padronizada }=\frac{(\text { população padrão na faixa etária })}{\left.\sum \text { (população padrão }\right)} \\
& \text { 0-19 anos taxa truncada }
\end{aligned}
$$

A análise das tendências das taxas de internação e mortalidade foi realizada pelo programa estatístico Joinpoint versão 4.5.0.1. Esse modelo de regressão ajusta os dados de uma série a partir do menor número possível de joinpoints (zero, ou seja, uma reta sem pontos de inflexão) e testa se a inclusão de mais joinpoints é estatisticamente significativa, identificando tendências de aumento ou declínio de taxas. Considerando um valor de $\alpha=0,05$ e intervalo de confiança (IC) de $95 \%$, uma tendência de aumento estatisticamente significativa ocorre se o limite inferior do IC estiver acima de zero ${ }^{19}$.

O estudo obedeceu às normas da Resolução 466/2012 e foi submetido e aprovado pelo Comitê de Ética e Deontologia em Estudos e Pesquisa da Universidade Federal do Vale do São Francisco (Univasf) sob CAAE 43973015.7.0000.5196.

\section{RESULTADOS}

$\mathrm{O}$ município de Petrolina $(\mathrm{PE})$ localiza-se às margens do rio São Francisco, na mesorregião do São Francisco Pernambucano, com território de $4572,76 \mathrm{Km}^{2}$ e população de 293.962 habitantes (censo 2010), sendo 112.359 (38,22\%) na faixa etária de 0 a 19 anos $^{20}$. Faz divisa com o município de Juazeiro (BA), que ocupa território de $6.454,25 \mathrm{Km}^{2}$, com população total de 197.965 habitantes (censo 2010), com 74.603 (37,6\%) menores de 19 anos $^{21}$. A distribuição da população na faixa etária de 0 a 19 anos é semelhante em ambos os municípios (Figura 1).

As taxas padronizadas de internação no município de Petrolina mantiveram-se mais elevadas que as de Juazeiro (Figura 2). Houve tendência de aumento significativa de internação por câncer de crianças e adolescentes em ambos os municípios (Tabela 1).

Houve 1.056 internações por câncer em Petrolina e 183 em Juazeiro (2004 a 2013). As internações por leucemia foram mais frequentes em Petrolina (33,5\%; $\mathrm{n}=354)$ e em Juazeiro $(52,4 \%$; $\mathrm{n}=175)$, seguidas de linfomas em Petrolina $(4,8 \% ; n=51)$

\section{Pirâmide de faixa etária e sexo (0-19 anos) Petrolina-PE 2010}

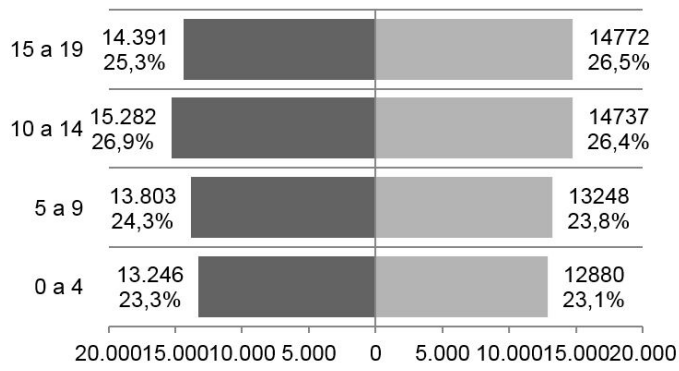

\section{Pirâmide de faixa etária e sexo (0-19 anos)} Juazeiro-BA 2010

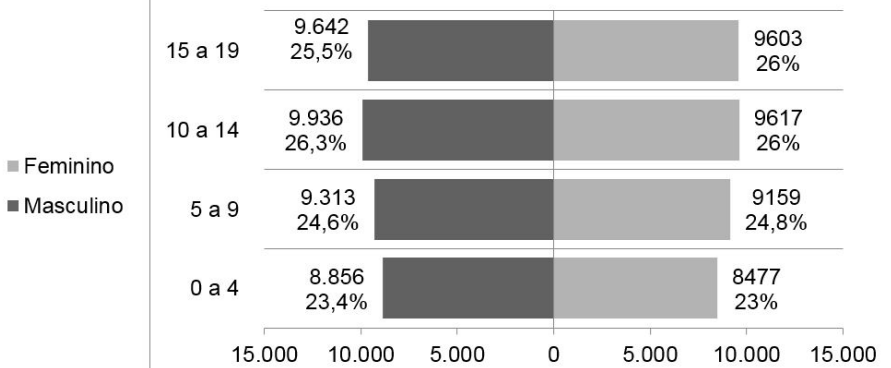

Feminino

-Masculino

Figura 1. Distribuição da população de 0 a 19 anos, por sexo e faixa etária, Juazeiro (BA) e Petrolina (PE), censo $2010^{20,21}$ 
e em Juazeiro $(9,8 \%$; $n=18)$. Ocorreu percentual maior de internações por leucemias no sexo masculino em Petrolina $(50,5 \% ; n=179)$ e em Juazeiro $(54 \% ; n=52)$. Nas duas cidades, o total de internações por câncer ocorreu em sua maioria no sexo masculino $(54,2 \% ; n=672)$.

No município de Petrolina, a maioria das internações por todos os tipos de câncer ocorreu na faixa etária de 1 a 4 anos $(27 \% ; n=285)$, seguidas pelas de 15 a 19 anos $(26,7 \% ; n=272)$.

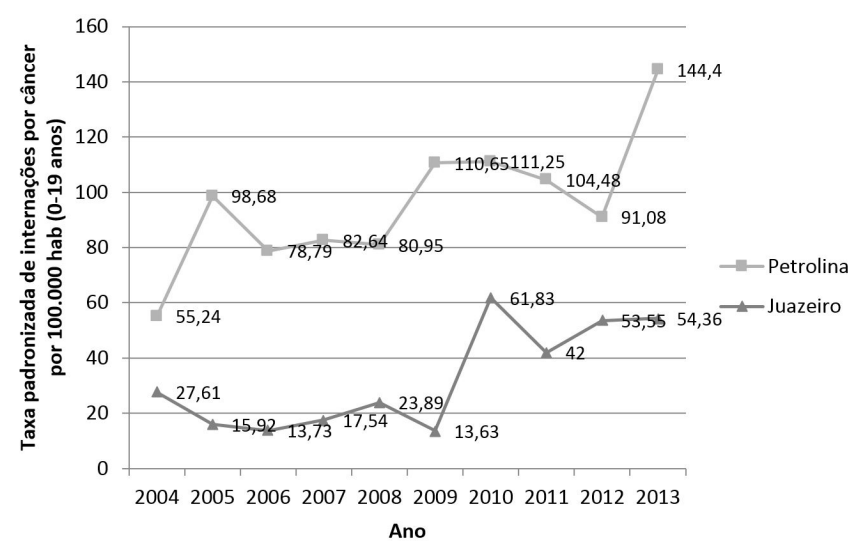

Figura 2. Taxa padronizada* de internações por câncer por 100.000 (0 a 19 anos), Juazeiro (BA) e Petrolina (PE), 2004 a 2013. Datasus Sistema de Informações Hospitalares do SUS (SIH/SUS $\left.{ }^{9}\right)$. ${ }^{*}$ População padrão mundial, modificada por Doll et al. ${ }^{18}$

Tabela 1. Tendência das taxas de internação e de mortalidade por neoplasias (0 a 19 anos) em Juazeiro (BA) e Petrolina (PE), de 2004 a 2013

\begin{tabular}{ccccccc}
\multirow{2}{*}{ Município } & \multicolumn{2}{c}{ Taxas de internação } & & \multicolumn{2}{c}{ Taxas de mortalidade } \\
\cline { 2 - 3 } \cline { 5 - 6 } & APC $^{\mathbf{a}}$ & IC95\% & & & APC & IC95\% $^{\mathbf{b}}$ \\
Juazeiro & $15,2^{*}$ & $2,9-29,0$ & & 38,1 & $-9,6-111,1$ \\
Petrolina & $6,7^{\star}$ & $1,9-11,7$ & & 10,5 & $-1,1-23,3$ \\
\hline
\end{tabular}

${ }^{a}$ Variação percentual anual; ${ }^{b}$ Intervalo de confiança de $95 \%$; ${ }^{*} \mathrm{p}$ Valor $<0,05$
Em Juazeiro, houve mais frequência de internações de 1 a 4 anos $(34,4 \% ; \mathrm{n}=63)$ e de 5 a 9 anos $(26,7 \% ; \mathrm{n}=49)$.

A leucemia foi mais frequente em Petrolina nas faixas etárias de 1 a 4 anos $(39,8 \% ; n=141)$ e 5 a 9 anos $(30,7 \%$; $=109)$, e em em Juazeiro, de 1 a 4 anos $(36,4 \%$; $=35)$ e de 5 a 9 anos $(33,3 \% ; \mathrm{n}=32)$.

A taxa de mortalidade padronizada pela população mundial por câncer apresentou oscilações com períodos de queda e de aumento nas duas cidades, destacando-se o ano de 2012 com as maiores taxas. Em Petrolina, as taxas alcançaram valores maiores que o dobro das ocorridas em Juazeiro (Tabela 2). Após a análise de tendências, verificou-se que o aumento dessas taxas de mortalidade não foi considerado estatisticamente significante nos dois municípios (Tabela 1).

Ocorreram 56 óbitos por câncer em Petrolina e 21 em Juazeiro. Os óbitos por leucemia foram mais frequentes em Petrolina $(n=21)$ e em Juazeiro $(n=9)$, seguidos de neoplasias do SNC em Petrolina $(n=13)$ e em Juazeiro $(n=2)$. O maior número de óbitos por leucemia ocorreu no sexo feminino $(n=9)$ em Juazeiro e no sexo masculino $(n=12)$ em Petrolina. Em sua maioria, os óbitos por leucemia ocorreram no sexo masculino $(\mathrm{n}=16)$.

A maioria dos óbitos ocorreu em indivíduos de 15 a 19 anos em Juazeiro $(n=7)$ e Petrolina $(n=21)$. Já os óbitos por leucemia foram mais frequentes na faixa etária de 5 a 9 anos no município de Juazeiro $(n=3)$ e de 15 a 19 anos em Petrolina $(n=8)$.

\section{DISCUSSÃO}

Neste estudo houve diferenças entre as taxas de internação em crianças e adolescentes para as neoplasias malignas em Petrolina e Juazeiro, com tendência de aumento significativo em ambos os municípios. Destacam-se as maiores taxas de internamento no município de Petrolina. Ressalta-se que em

Tabela 2. Taxa de mortalidade por câncer, bruta e padronizada para idade, por 1.000 .000 de habitantes (0 a 19 anos), Juazeiro (BA) e Petrolina (PE), 2004 a 2013. Datasus - Sistema de Informações sobre Mortalidade (SIM)

\begin{tabular}{|c|c|c|c|c|c|c|}
\hline & \multicolumn{3}{|c|}{ Juazeiro } & \multicolumn{3}{|c|}{ Petrolina } \\
\hline & $\begin{array}{l}\text { Taxa } \\
\text { bruta }\end{array}$ & $\begin{array}{l}\text { Taxa padronizada da } \\
\text { população no Brasil }\end{array}$ & $\begin{array}{l}\text { Taxa padronizada da } \\
\text { população mundial }\end{array}$ & $\begin{array}{c}\text { Taxa } \\
\text { bruta }\end{array}$ & $\begin{array}{l}\text { Taxa padronizada da } \\
\text { população no Brasil }\end{array}$ & $\begin{array}{l}\text { Taxa padronizada da } \\
\text { população mundial }\end{array}$ \\
\hline \multicolumn{7}{|l|}{ Ano } \\
\hline 2004 & 11,7 & 12,1 & 10,7 & 18,5 & 19,1 & 16,7 \\
\hline 2005 & 11,1 & 11,6 & 9,9 & 35,3 & 36,4 & 32,7 \\
\hline 2006 & - & - & - & 51,6 & 52,1 & 51 \\
\hline 2007 & 23 & 23 & 22,9 & 64,2 & 64,5 & 58,6 \\
\hline 2008 & 20,9 & 21,8 & 19,2 & 35,8 & 35,9 & 35,9 \\
\hline 2009 & 30,9 & 29,3 & 33,8 & 80 & 80 & 78,9 \\
\hline 2010 & 26,8 & 25,6 & 30,8 & 62,3 & 61,2 & 68,1 \\
\hline 2011 & 39,8 & 38,7 & 45,7 & 39 & 35,4 & 33,2 \\
\hline 2012 & 79 & 78,8 & 77,8 & 85,8 & 84,2 & 92,8 \\
\hline 2013 & 26,3 & 27,4 & 22,7 & 51,4 & 51,2 & 50,8 \\
\hline
\end{tabular}

* População do Brasil, segundo o IBGE 2 , para cada ano. ${ }^{* *}$ População padrão mundial, modificada por Doll et al. ${ }^{18}$ 
Petrolina há um serviço de Oncologia Pediátrica, implantado desde 2011, que é referência para a Rede Interestadual de Saúde (PE/BA), fato este que pode ter contribuído para o maior acesso ao serviço especializado e consequente aumento do número de casos de internação.

Este estudo demonstrou que a leucemia é o câncer com maior número de internações entre jovens menores de 19 anos, seguido por linfomas e tumores do SNC, perfil que corrobora os resultados encontrados no Brasil e em outros países ${ }^{1,7,22}$. Dados do Instituto Nacional de Câncer (Inca) descrevem que as neoplasias mais incidentes entre os jovens atendidos em unidades hospitalares são as leucemias (30,6\%), seguidas de linfomas $(16,6 \%)$ e tumores do SNC $(9,6 \%)^{23}$.

O maior número de internações em indivíduos do sexo masculino e na faixa etária de 1 a 4 anos foi de leucemia linfoide aguda e coincide com a literatura. No estado do Mato Grosso, 53,7\% dos casos de câncer ocorreram em meninos e as leucemias foram as mais incidentes ${ }^{22}$. Um estudo feito em Ribeirão Preto (SP) descreveu que o maior número de internações por câncer ocorreu por leucemia linfoide aguda, no sexo masculino, na faixa etária de 1 a $4 \operatorname{anos}^{24}$. No Brasil, $32,4 \%$ das crianças internadas diagnosticadas com leucemia estão na faixa etária de 1 a 4 anos $^{23}$. Na maioria dos países do mundo, as crianças com menos de 5 anos de idade são as mais acometidas por leucemia ${ }^{1,25}$.

Os fatores de risco conhecidos para a leucemia linfoide aguda, câncer mais comum na infância, são os polimorfismos genéticos em CYP2E1, GSTM1, NQO1, NAT2, MDR1 e XRCC1 que aumentam o risco dessa neoplasia, quando associado a exposições ambientais, como agrotóxicos e inseticidas domésticos, tabagismo, trialometanos, consumo de álcool e raios $\mathrm{X}^{26}$. No tocante à questão da exposição ambiental, deve-se levar em conta que Petrolina e Juazeiro são polos de fruticultura irrigada onde há utilização indiscriminada de agrotóxicos, principalmente de organofosforados ${ }^{13}$. Crianças e adolescentes que vivem em áreas de cultivo agrícola ou com familiares que trabalhem com produtos químicos apresentam mais risco de contaminação no ambiente domiciliar.

Essa população possui vulnerabilidades específicas, como intoxicação por agrotóxicos, contaminação da dieta e hábitos comportamentais. Por exemplo, o hábito de levar as mãos à boca eleva o risco de contaminação; a ingestão acidental de veneno pode ser mais tóxica devido à menor superfície corporal da criança; acrescenta-se o fato de os pais trabalharem em lavouras e sempre trazerem para dentro de casa roupas ou equipamentos contaminados; assim como a exposição ocupacional da mãe durante a gestação pode aumentar o risco de adoecimento por leucemia ${ }^{23,27,28}$.

As taxas de mortalidade por câncer infantojuvenil tiveram um aumento em ambos os municípios, diferentemente do encontrado em outros países que descrevem tendência de queda da mortalidade, principalmente nos desenvolvidos. No Brasil, um estudo que avaliou as taxas de mortalidade por câncer na faixa etária de 0 a 14 anos, no período de 1979 a 2008, identificou que houve uma diminuição significativa dessas taxas em crianças com leucemia, porém com diferenças entre as regiões brasileiras. A mortalidade por cânceres infantojuvenis nas regiões Sul, Sudeste e Centro-Oeste reduziu de 0,5\% a 1,5\% ao ano, porém a mortalidade aumentou no Norte e Nordeste de $2 \%$ a $3 \%$ ao ano ${ }^{29}$. Outro estudo descreve que no Brasil, no período de 1980 a 2010, as taxas de mortalidade por leucemias linfoides e mieloides apresentam tendência de crescimento, com exceção das leucemias linfoides entre meninos menores de 4 anos de idade ${ }^{30}$.

Entre os fatores que podem estar associados ao aumento da mortalidade em estados do Nordeste do Brasil, há o número reduzido de serviços de oncologia e médicos oncologistas ${ }^{7,31}$. Como o atendimento de oncologia pediátrico foi implantado na região em 2011, a população infantojuvenil conviveu durante muito tempo com a dificuldade de acesso a serviços especializados, o que pode ter contribuído para o diagnóstico tardio e o aumento de mortalidade aqui descrita.

Nos Estados Unidos, a taxa de mortalidade por câncer infantil reduziu de 49/ 1.000.000 em 1975 para 22/1.000.000 em 2008 ${ }^{1,7}$. Na América Latina, houve tendência de aumento nas taxas de mortalidade por leucemia no México, Equador e Colômbia e declínio na Argentina e Costa Rica ${ }^{32}$.

As taxas de mortalidade por neoplasias malignas de 60/1.000.0000 ( 0 a 19 anos), em ambos os municípios, encontram-se acima das taxas de mortalidade brasileiras $(44,22 / 1.000 .000)$ e das taxas de mortalidade do Nordeste $(43,46 / 1.000 .000)^{23}$. Taxas acima de 60/1.000.000 foram descritas no sexo masculino em países como Cuba, Venezuela, Cingapura, Azerbaijão, Cazaquistão e Uzbequistão ${ }^{33}$. Essas altas taxas de mortalidade encontradas em Petrolina e Juazeiro devem ser analisadas com cuidado, pois podem estar influenciadas pela qualidade do diagnóstico, das declarações de óbito e pelo acesso a serviços de oncologia pediátrica ${ }^{29}$. Como o número de óbitos é pequeno, uma ínfima variação no número absoluto também pode provocar instabilidade das taxas de mortalidade ${ }^{34}$. Além disso, uma correção da população do Instituto Brasileiro de Geografia e Estatística (IBGE) no censo de 2010, no município de Juazeiro, reduziu o número de habitantes de 0 a 19 anos de 97.065 , em 2009, para 74.603 , em 2010, o que pode ter influenciado nas taxas de mortalidade ${ }^{35}$.

Nesse estudo, a leucemia foi responsável pelo maior número de óbitos, seguida pelos tumores do SNC, situação semelhante a que ocorre no Brasil, onde houve mais frequência de óbitos por leucemia $(32,9 \%)$, seguida de neoplasias do SNC $(20 \%)^{23}$. As leucemias linfoides agudas também são a principal causa 
de mortalidade por câncer em outros países do mundo, como Chile, Canadá, Estados Unidos, Suíça e Japão ${ }^{33,36,37}$.

Em Petrolina e Juazeiro, a mortalidade foi maior no sexo masculino, semelhantemente a resultados do estado do Mato Grosso (54,9\%) e de Ribeirão Preto (SP) ${ }^{22,24}$. Maiores taxas de mortalidade entre os meninos por leucemia foram verificadas no Brasil, Japão, nas Américas, Ásia e Oceania ${ }^{23,30,33,37}$.

Esse estudo mostrou que a faixa etária com maior número de óbitos foi a de 15 a 19 anos. No Brasil, no período de 2009 a 2013, os óbitos por leucemia foram mais incidentes na faixa etária de 15 a 19 anos (27,5\%), 10 a 14 anos $(25,6 \%)$ e 5 a $9(23,7 \%)^{23}$.

As diferenças de mortalidade por faixa etária podem estar associadas ao diagnóstico tardio. Crianças mais velhas têm mais risco de diagnóstico tardio, o que pode estar associado às características do tumor e ao fato de as crianças mais jovens serem mais dependentes de cuidados dos pais, o que facilitaria a identificação de sinais e sintomas mais precoces ${ }^{6}$.

Os resultados deste estudo descrevem tendência significativa de aumento das taxas de internação e de aumento das taxas de mortalidade por neoplasias em crianças e adolescentes, em Petrolina e Juazeiro. As taxas de mortalidade foram mais altas do que as encontradas no Brasil e na região Nordeste. Observou-se, ainda, maior número de internações e maior taxa de mortalidade no município de Petrolina comparados aos de Juazeiro nos dez anos do estudo (2004-2013).

Entre as limitações do estudo, há o uso de dados secundários de sistemas de informação que podem estar incompletos, ter codificação incorreta e não permitir identificar os fatores de risco associados ao perfil de morbimortalidade. Além disso, para avaliar a morbidade, foi usado o Sistema de Internações Hospitalares do Sistema Único de Saúde (SIH/SUS), que não é uma medida direta do adoecimento, visto que apenas reflete os indivíduos internados pela doença.

As tendências significativas de aumento das taxas de morbidade e as taxas de mortalidade acima de 60/1.000.000 (0 a 19 anos) necessitam de mais investigação e podem estar relacionadas à melhora no acesso ao serviço especializado de oncologia pediátrica, ao diagnóstico tardio e à exposição ambiental a agrotóxicos. O conhecimento desses fatores é fundamental para haver implementação de políticas públicas intersetoriais para reduzir as vulnerabilidades e a morbimortalidade por câncer na população infantojuvenil nessa região.

\section{REFERÊNCIAS}

1. Braga PE, Latorre MRDO, Curado MP. Câncer na infância: análise comparativa da incidência, mortalidade e sobrevida em Goiânia (Brasil) e outros países. Cad Saude Publica. 2002;18(1):33-44. http://dx.doi.org/10.1590/S0102311X2002000100004. PMid:11910422.

2. Instituto Nacional de Câncer José Alencar Gomes da Silva. Coordenação de Prevenção e Vigilância. Estimativa 2014: incidência de câncer no Brasil. Rio de Janeiro: INCA; 2014.

3. Carpenter DO, Bushkin-bedient S. Exposure to chemicals and radiation during childhood and risk for cancer later in life. J Adolesc Health. 2013;52(5 Suppl):S21-9. http://dx.doi.org/10.1016/j.jadohealth.2013.01.027. PMid:23601608.

4. Cha ES, Kong KE, Moon EK, Khang Y, Lee WJ. Childhood cancer mortality and birth characteristics in Korea: a national population-based birth cohort study. J Korean Med Sci. 2011;26(3):339-45. http://dx.doi.org/10.3346/ jkms.2011.26.3.339. PMid:21394300.

5. Spector LG, Ross JE, Olshan AF. Children's Oncology Group's 2013 blueprint for research: epidemiology. Pediatr Blood Cancer. 2013;60(6):1059-62. PMid:23255344.

6. Dang-tan T, Franco EL. Diagnosis delays in childhood cancer. Cancer. 2007;110(4):703-13. http://dx.doi.org/10.1002/cncr.22849. PMid:17620277.

7. Siegel R, Naishadham D, Jemal A. Cancer statistics. CA Cancer J Clin. 2012;62(1):10-29. http://dx.doi.org/10.3322/caac.20138. PMid:22237781.

8. Instituto Nacional de Câncer José Alencar Gomes da Silva. Diagnóstico precoce do câncer na criança e no adolescente. 2. ed. Rio de Janeiro: INCA; 2014
9. Departamento de Informática do Sistema Único de Saúde. Mortalidade Brasil: óbitos por residência por faixa etária segundo capítulo CID-10 Período: 2014 [Internet]. Brasília: DATASUS; 2017 [citado em 2017 jan 18]. Disponível em: http://tabnet.datasus.gov.br/cgi/tabcgi.exe?sim/cnv/ obt10uf.def

10. Instituto Nacional de Câncer José Alencar Gomes da Silva. ABC do câncer: abordagens básicas para o controle do câncer. Rio de Janeiro: INCA; 2011.

11. Grabois MF, Oliveira EXG, Carvalho MS. Assistência ao câncer entre crianças e adolescentes: mapeamento dos fluxos origem-destino no Brasil. Rev Saude Publica. 2013;47(2):368-78. http://dx.doi.org/10.1590/S00348910.2013047004305. PMid:24037365.

12. Bustamante PMAC. A fruticultura no Brasil e no Vale do São Francisco: vantagens e desafios. Rev Econ NE. 2009;40(1):154-71.

13. Bedor CNG, Ramos LO, Pereira PJ, Rego MAV, Pavão AC, Augusto LG. Vulnerabilidades e situações de riscos relacionados ao uso de agrotóxicos na fruticultura irrigada. Rev Bras Epidemiol. 2009;12(1):39-49. http:// dx.doi.org/10.1590/S1415-790X2009000100005.

14. Leite MNP, Bedor CNG. Análise temporal da mortalidade por câncer no submédio do Vale do São Francisco. In: Anais da Jornada de Iniciação Científica da UNIVASF [Internet]; 2007; Petrolina. Petrolina: UNIVASF; 2007 [citado em 2016 dez 18]. Disponível em: http://www.prppg.univasf. edu.br/?p=jornada

15. Bastos LDM. Caracterização clínico-epidemiológica da mortalidade por neoplasia maligna em trabalhadores rurais do Vale do São Francisco [Trabalho de Conclusão]. Petrolina (PE): Universidade Federal do Vale do São Francisco; 2011. 
16. Moura LTR. Perfil clínico-epidemiológico dos trabalhadores acometidos por câncer atendidos em um centro de oncologia do submédio do Vale do São Francisco [dissertação]. Petrolina (PE): Universidade Federal do Vale do São Francisco; 2014.

17. Associação Petrolinense de Aparo à Maternidade e Infância. Nosso trabalho [Internet]. 2012 [citado em 2016 dez 20]. Disponível em: http://apami.org. br/nosso-trabalho/

18. Doll R, Payne P, Waterhouse J, editors. Cancer incidence in five continents: a technical report. Berlin: Springer-Verlag; 1966. http://dx.doi.org/10.1007/9783-642-85849-9.

19. Kim HJ, Fay MP, Feuer EJ, Midthune DN. Permutation tests for joinpoint regression with applications to cancer rates. Stat Med. 2000;19(3):335-51. PMID: 10649300.

20. Instituto Brasileiro de Geografia e Estatística. Petrolina infográficos evolução populacional e pirâmide etária [Internet]. 2017 [citado em 2017 jul 13]. Disponível em: http://cidades.ibge.gov.br/painel/populacao. php?codmun $=261110$

21. Instituto Brasileiro de Geografia e Estatística. Juazeiro infográficos evolução populacional e pirâmide etária [Internet]. 2017 [citado em 2017 jul 13]. Disponível em:http://ibge.gov.br/cidadesat/painel/populacao.php?lang=\& codmun=291840\&search=bahia\%7Cjuazeiro\%7Cinfograficos:-evolucaopopulacional-e-piramide-etaria

22. Curvo HRM, Pignati WA, Pignatti MG. Morbimortalidade por câncer infantojuvenil associada ao uso agrícola de agrotóxicos no Estado de Mato Grosso, Brasil. Cad Saude Colet. 2013;21(1):10-7. http://dx.doi.org/10.1590/ S1414-462X2013000100003.

23. Instituto Nacional de Câncer José Alencar Gomes da Silva. Coordenação de Prevenção e Vigilância. Incidência, mortalidade e morbidade hospitalar por câncer em crianças, adolescentes e adultos jovens no Brasil: informações dos registros de câncer e do sistema de mortalidade. Rio de Janeiro: INCA; 2016.

24. Pan R, Marques AR, Costa ML Jr, Nascimento LC. Caracterização das internações hospitalares de crianças e adolescentes com neoplasias. Rev Lat Am Enfermagem. 2011;19(6):1413-20. http://dx.doi.org/10.1590/ S0104-11692011000600019. PMid:22249677.

25. Steliarova-Foucher E, Colombet M, Ries LAG, Moreno F, Dolya A, Bray F, et al. International incidence of childhood cancer, 2001-10: a populationbased registry study. Lancet Oncol. 2017;18(6):719-31. http://dx.doi. org/10.1016/S1470-2045(17)30186-9. PMid:28410997.

26. Brisson GD, Alves LR, Pombo-de-Oliveira MS. Genetic susceptibility in childhood acute leukaemias: a systematic review. Ecancermedicalscience. 2015;9(539):1-28. http://dx.doi.org/10.3332/ecancer.2015.539. PMid:26045716.
27. Ferreira JD, Couto AC, Pombo de Oliveira MS, Koifman S. In utero pesticide exposure and Leukemia in Brazilian children $>2$ years of age. Environ Health Perspect. 2013;121(2):269-75. PMid:23092909.

28. Roberts JR, Karr KJ. Pesticide exposure in children. Pediatr. 2012;130(6):176588. http://dx.doi.org/10.1542/peds.2012-2758. PMid:23184105.

29. Ferman S, Santos MO, Ferreira JM, Reis RS, Oliveira JF, Pombo-de-Oliveira MS, et al. Childhood cancer mortality trends in Brazil, 1979-2008. Clinics. 2013;68(2):219-24. http://dx.doi.org/10.6061/clinics/2013(02)OA16. PMid:23525319.

30. Silva FF, Zandonade E, Zouain-Figueiredo GP. Analysis of childhood leukemia mortality trends in Brazil, from 1980 to 2010. J Pediatr. 2014;90(6):587-92. http://dx.doi.org/10.1016/j.jped.2013.12.013. PMid:24950473.

31. Ribeiro KB, Lopes LF, Camargo B. Trends in childhood leukemia mortality in Brazil and correlation with social inequalities. Cancer. 2007;110(8):182331. http://dx.doi.org/10.1002/cncr.22982. PMid:17786938.

32. Curado MP, Pontes T, Guerra-yi ME, Camargo Cancela M. Leukemia mortality trends in children, adolescents, and young adults in Latin America. Rev Panam Salud Publica. 2011;29(2):96-102. http://dx.doi.org/10.1590/ S1020-49892011000200004. PMid:21437366.

33. Chatenoud L, Bertuccio P, Bosetti C, Levi F, Negri F, La Vecchia C. Childhood cancer mortality in America, Asia, and Oceania, 1970 through 2007. Cancer. 2010;116(21):5063-74. http://dx.doi.org/10.1002/cncr.25406. PMid:20629033.

34. Boing AF, Boing AC. Mortalidade infantil por causas evitáveis no Brasil: um estudo ecológico no período 2000-2002. Cad Saude Publica. 2008;24(2):44755. http://dx.doi.org/10.1590/S0102-311X2008000200024. PMid:18278292.

35. Departamento de Informática do Sistema Único de Saúde. População Residente - Bahia, população residente por ano e faixa etária 0 a 19 anos, Município: Juazeiro, 2004-2012 [Internet]. Brasília: DATASUS; 2017 [citado em 2017 ago 7]. Disponível em: http://tabnet.datasus.gov.br/cgi/tabcgi. exe?ibge/cnv/popba.def

36. Schindler M, Spycher BD, Ammann RA, Ansari M, Michel G, Kuehni CE. Cause-specific long-term mortality in survivors of childhood cancer in Switzerland: a population-based study. Int J Cancer. 2016;139(2):322-33. http://dx.doi.org/10.1002/ijc.30080. PMid:26950898.

37. Yang L, Fujimoto J. Childhood cancer mortality in Japan, 1980-2013. BMC Cancer. 2015;15(446):1-8. http://dx.doi.org/10.1186/s12885-015-1472-x. PMid:26026605.
Recebido em: Dez. 29, 2017 Aceito em: Fev. 22, 2018 